\title{
Final report: Levitated Dipole Experiment
}

\author{
J. Kesner \\ MIT Plasma Science and Fusion Center \\ Cambridge, MA 02139 \\ M. Mauel \\ Department of Applied Physics, Columbia University \\ New York, NY 10027 \\ Submitted to the U.S. Department of Energy \\ Grants DE-FG02-98ER54458/9
}

March 10, 2013

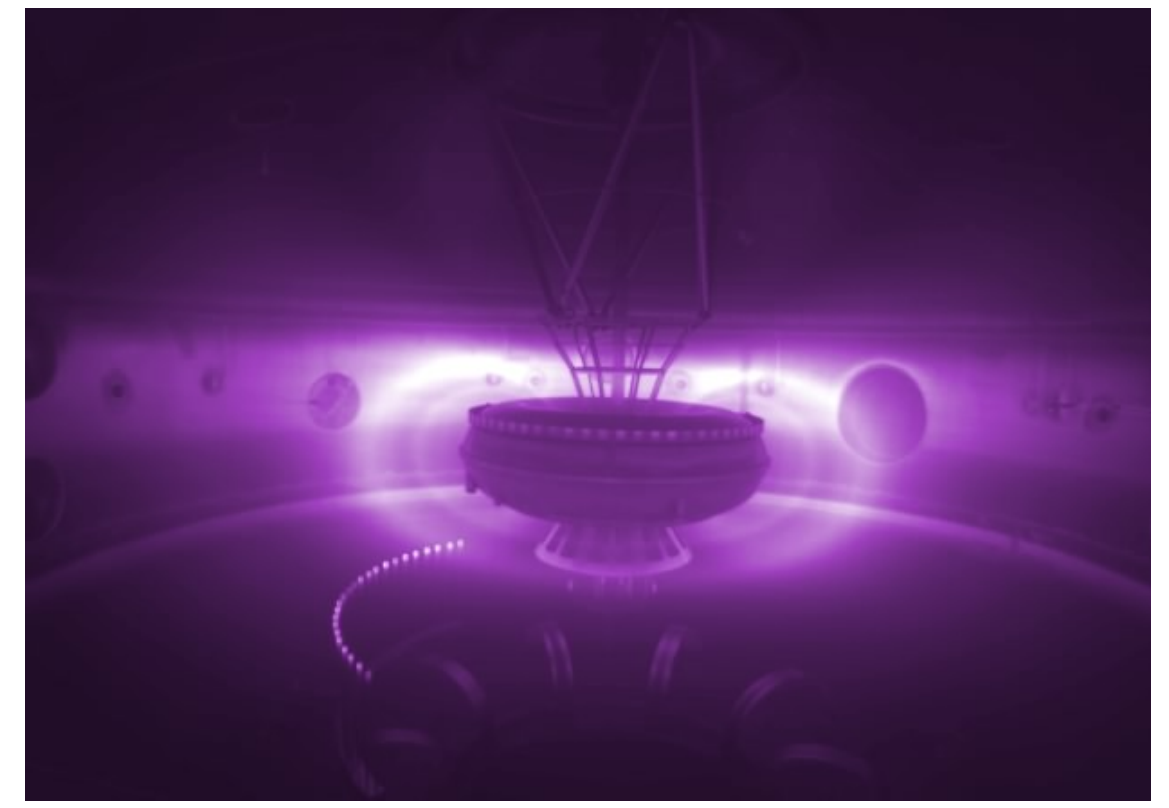




\title{
Final report: Levitated Dipole Experiment
}

\author{
J. Kesner (MIT, Plasma Science and Fusion Center) \\ M. Mauel (Columbia University, Department of Applied Physics) \\ Plasma Science and Fusion Center, MIT, Cambridge, MA \\ J. Kesner 〈mailto: kesner@psfc.mit.edu〉
}

Columbia University, Dept. of Applied Physics and Applied Math, New York, NY

M. Mauel 〈mailto: mauel@columbia.edu〉

Grants DE-FG02-98ER54458/9

DOE Office of Fusion Energy Science

\section{Research Accomplishments}

Since the very first experiments with the LDX, research progress was rapid and significant. Initial experiments were conducted with the high-field superconducting coil suspended by three thin rods. These experiments produced long-pulse, quasi-steady-state microwave discharges, lasting more than $10 \mathrm{~s}$, having peak beta values of 20\% [1]. High-beta, near steady-state discharges have been maintained in LDX for more than 20 seconds, and this capability makes LDX the longest pulse fusion confinement experiment now operating in the U.S. fusion program. In both supported and levitated configurations, detailed measurements are made of discharge evolution, plasma dynamics and instability, and the roles of gas fueling, microwave power deposition profiles, and plasma boundary shape. High-temperature plasma is created by multifrequency electron cyclotron resonance heating allowing control of heating profiles. Depending upon neutral fueling rates, the LDX discharges contain a fraction of energetic electrons, with mean energies above $50 \mathrm{keV}$. Depending on whether or not the superconducting dipole is levitated or supported, the peak thermal electron temperature is estimated to exceed $500 \mathrm{eV}$ and peak densities reach $10^{18} \mathrm{~m}^{-3}$.

Several significant discoveries resulted from the routine investigation of plasma confinement with a magnetically-levitated dipole. For the first time, toroidal plasma with pressure approaching the pressure of the confining magnetic field was well-confined in steady-state without a toroidal magnetic field. Magnetic levitation proved to be reliable and is now routine. The dipole's cryostat allows up to three hours of "float time" between re-cooling with liquid helium and providing scientists unprecedented access to the physics of magnetizd plasma. Levitation eliminates field-aligned particle sources and sinks and results in a toroidal, magnetically-confined plasma where profiles are determined by cross-field transport. We find levitation causes the central plasma density to increase dramatically and to significantly improve the confinement of thermal plasma. Several diagnostic systems have been used to measure plasma fluctuations, and these appear to represent low-frequency convection that may lead to adiabatic heating and strongly peaked pressure profiles.

These experiments are remarkable, and the motivate wide-ranging studies of plasma found in space and confined for fusion energy. In the following report, we describe: (i) observations of the centrally-peaked density profile that appears naturally as a consequence of a strong turbulent pinch, (ii) observations of overall density and pressure increases that suggest large improvements to the thermal electron confinement time result occur during levitation, and (iii) the remarkable properties of low-frequency plasma fluctuations that cause magnetized plasma to "self-organize" into well-confined, centrally-peaked profiles that are relative to fusion and to space. 


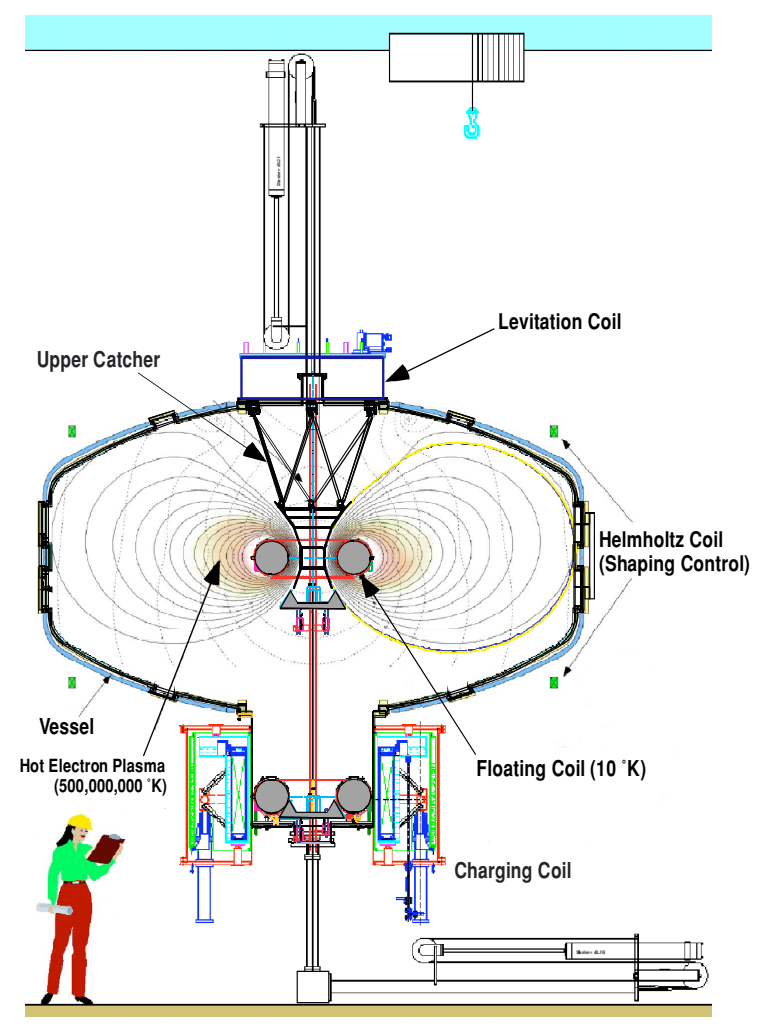

Figure 1: Schematic view of the LDX facility.

\section{Centrally Peaked Density during Levitation}

Measuring the evolution and establishment of plasma profiles is one of the most important tasks in the investigation of plasma confinement. Profile evolution and dynamics indicate underlying, and often very complex, dynamical and transport processes. In general, both field-aligned and cross-field processes are active within magnetized plasma; however, one often dominates over the other in the determination of plasma profiles. For example, plasma in the Earth's plasmasphere fills slowly over days from atmospheric sources along field-lines, while, outside the plasmasphere, fast convective flows perpendicular to the magnetic field predominate plasma dynamics, especially during magnetic storms when these flows are large $[2,3,4]$. In fusion confinement devices, the profiles within hot central region are determined by cross-field processes while the edge, "scrapeoff-layer", is dominated by field-aligned thermal and particle flow to material surfaces.

In the Levitated Dipole Experiment (LDX), we have been able to observe directly the changing roles of field-aligned and cross-field processes in the establishment of density profile. When the magnetic dipole floats, high-temperature plasma is confined within a large central confinement volume where magnetic field lines pass through the magnetic poles and never contact material surfaces. Within this central region, profiles are set entirely by cross-field processes. However, when we support the dipole mechanically, cross-field plasma mixing becomes relatively unimportant and very different plasma profiles result. The observation of central density peaking during levitation of the dipole is a significant discovery that supports the dipole fusion concept. 


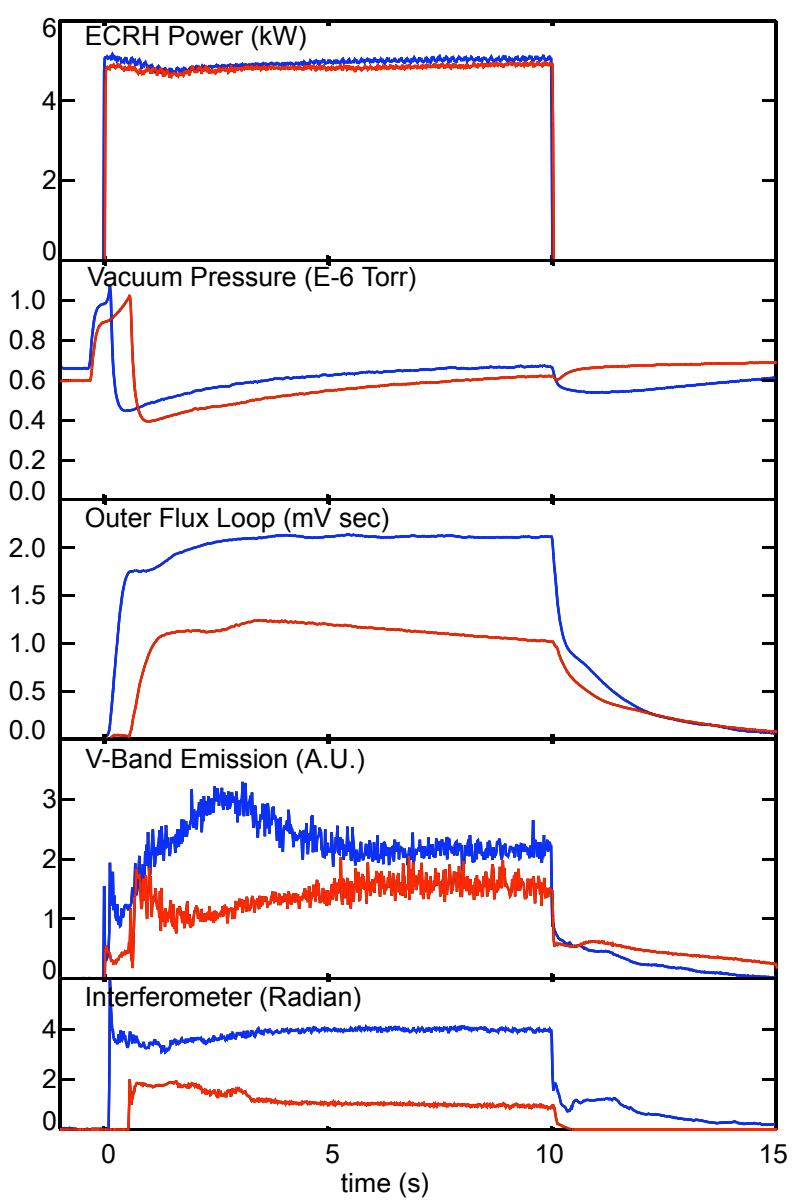

Levitated - S71213004

Supported— $\$ 71213003$

Levitated: Peaked Density Profile

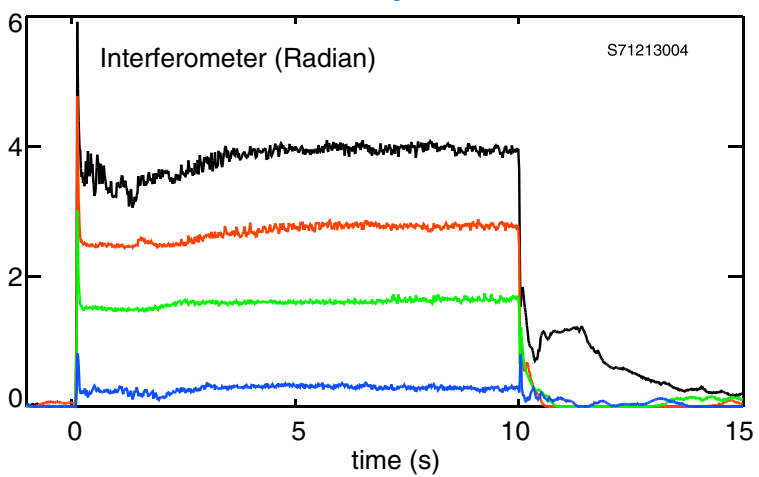

Supported: Uniform Density Profile

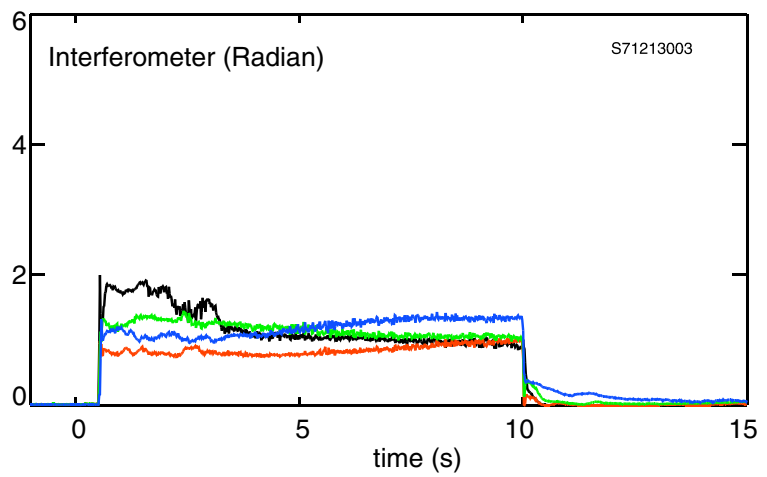

Figure 2: Measurement of the dramatic central peaking of the plasma density profile from one of the very first experiments with a levitated dipole. Two nearly equivalent discharges were created with $5 \mathrm{~kW}$ of long-pulse ECRH. When the dipole was supported mechanically (Shot 71213003), then the four interferometer cords measure nearly equivalent line density. When the dipole is levitated (Shot 71213004), then the central line density is much larger than the edge.

These experiments used a four-channel microwave interferometer array[5] to measure plasma density profile evolution. Whenever the dipole magnet is levitated, high-speed measurements of the plasma with the interferometer array show the development of centrally peaked density profiles that resemble the profiles observed during strong convection in active magnetospheres. In comparison, when the dipole magnet is mechanically suspended, the density profile is uniform and shows no evidence of central peaking. The central peaking of density is (i) a robust result, (ii) observed immediately from our very first experiments using a magnetically levitated dipole, (iii) appears at nearly all power levels and conditions where the hot electron interchange instability does not disturb discharge dynamics, (iv) occurs rapidly (within approximately $15 \mathrm{~ms}$ ), and (v) does not depend upon plasma beta.

LDX density profiles were measured using a four-channel microwave interferometer [5]. The upper levitation magnet creates magnetic field null that defines the outer boundary between closed and open field lines. The inner boundary is set by magnetic field lines that contact the floating superconducting dipole. Along the equatorial midplane, closed field lines extend from $0.68 \mathrm{~m}$ to 


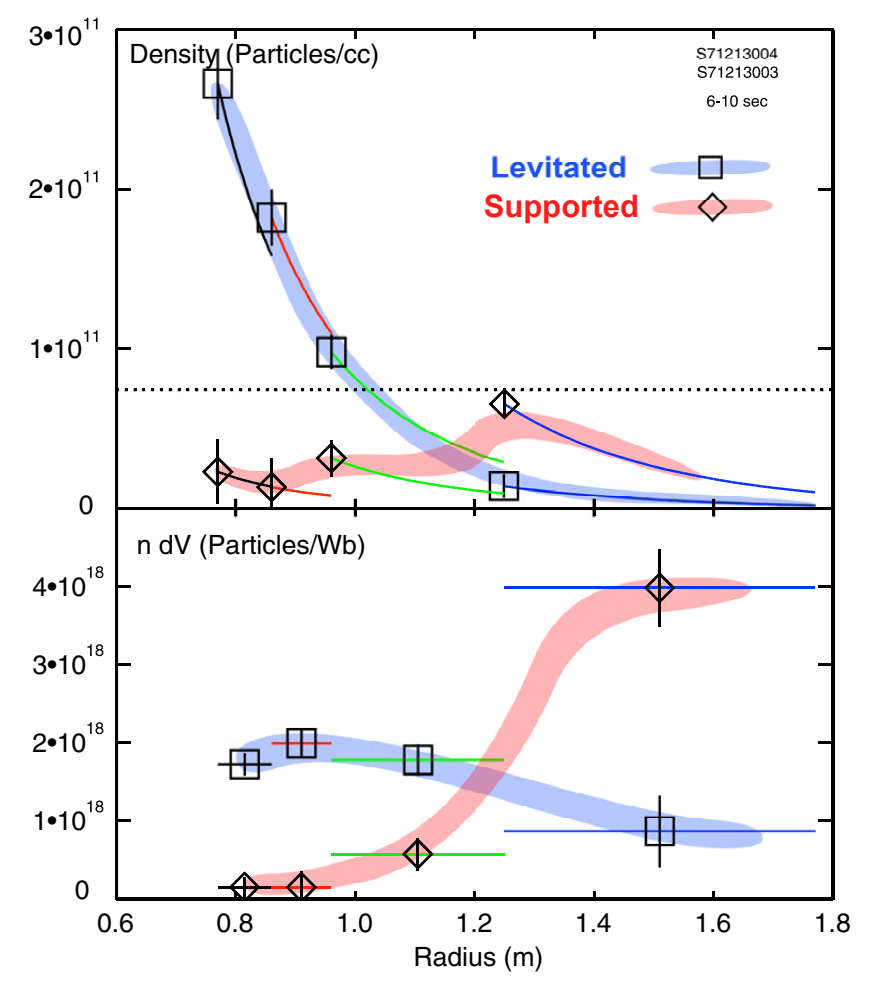

(a)

Deviation Parameter from $\delta(n V)=0$ Profile
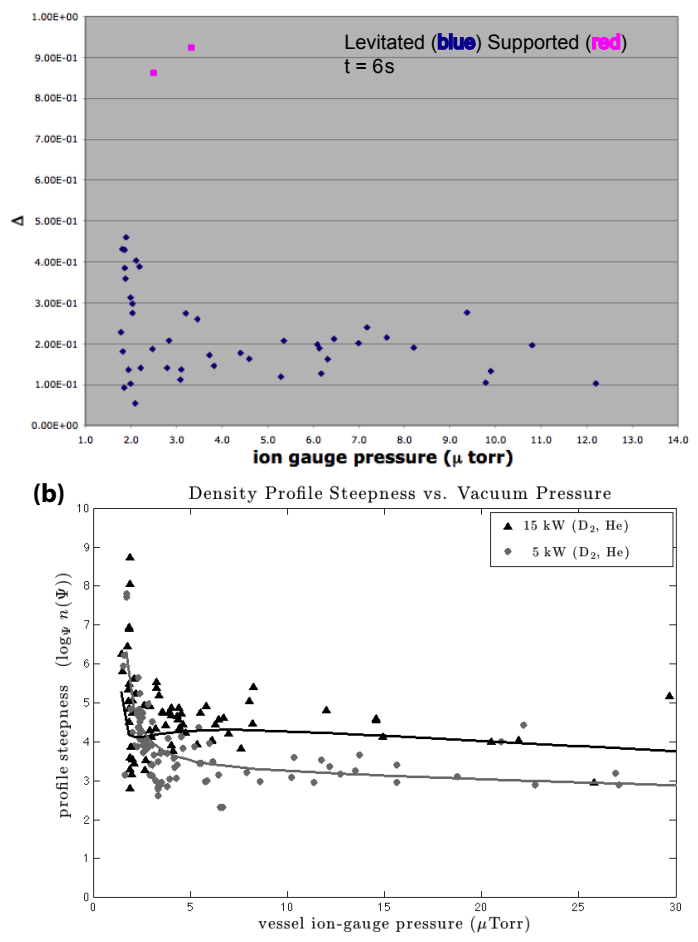

Figure 3: (Left) Reconstruction of the plasma density profile for the levitated and supported discharges shown in Fig. 6. Levitation results in factor-of-ten increase of central density that is characterized by approximately equal numbers of particles within tubes of equal magnetic flux and corresponds to an strong anomalous particle pinch. (Right) Parameters characterizing the density profile as a function of neutral gas fueling and atomic species and for several heating power levels. For all cases, except for low-pressures usually dominated by hot electron instability, the density profile is "stationary".

$1.75 \mathrm{~m}$. The LDX interferometer records line density over everything except the innermost region of closed field lines with radii of tangency of $0.77 \mathrm{~m}, 0.86 \mathrm{~m}, 0.96 \mathrm{~m}$ and $1.25 \mathrm{~m}$. The inner most interferometer channel is able to record the density inside the cyclotron resonance of the $2.45 \mathrm{GHz}$ heating source, and the inner three channels pass through the $2.45 \mathrm{GHz}$ upper hybrid resonance at typical density. These measurements can be inverted as a function of time to obtain equivalent density profiles based on any of several model parameterizations. These models can be further constrained by estimates of the edge plasma density obtained from Langmuir probes. Since only four cords are available, the reconstructed profiles are necessarily of limited spatial resolution and, moreover, the inversion process compounds the uncertainties towards the profile's central core. Nevertheless, the accuracy of the computed radial density profiles are sufficient to answer basic questions about the dynamics of LDX plasmas regarding the approximate value of the density and the relative steepness or broadness of the profile under different experimental conditions.

Fig. 2 illustrates two similar discharges that were prepared to be similar in every way except the dipole was supported mechanically in the first discharge (Shot 71213003) and magnetically in the second (Shot 71213004). Both discharges were created and sustained with $5 \mathrm{~kW}$ of multiplefrequency ECRH and had an equivalent magnetic geometry. Magnetic equilibrium reconstruction 

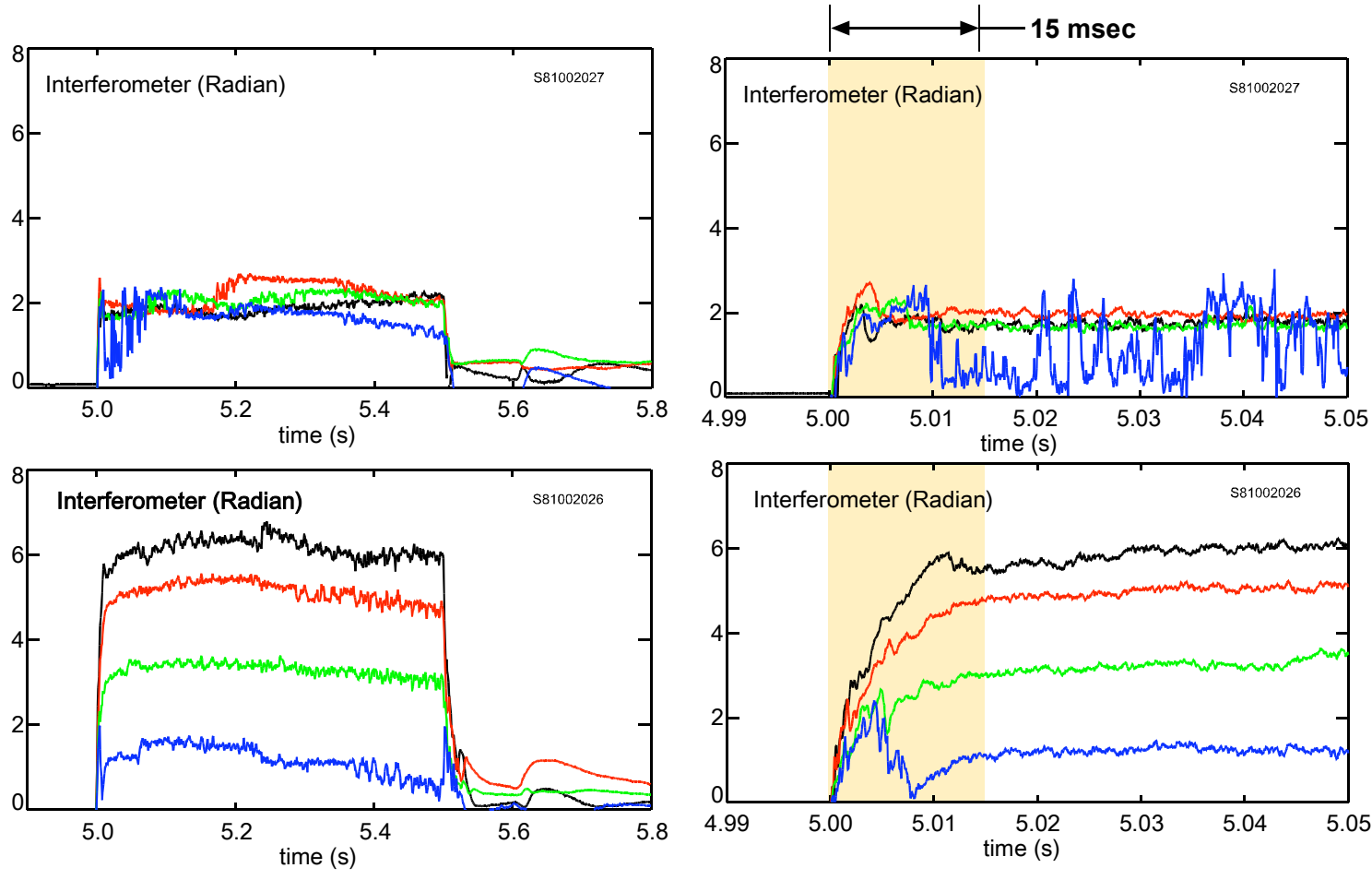

Figure 4: The plasma density profile is established on a short, $15 \mathrm{~ms}$, time-scale; whereas, the plasma energy time scale is much longer. In this figure, we show measurements during short $1.5 \mathrm{~s}$ heating pulses applied during similar supported and levitated cases. As shown in the close-up figure (right), during levitation, "stationary" density profile forms within $15 \mathrm{~ms}$, although the density during the initial $5 \mathrm{~ms}$ of heating are nearly equivalent.

shows the relationship between magnetic flux, plasma diamagnetic current, and stored plasma energy. For the plasma produced with a supported dipole, the plasma diamagnetic current reached $1.3 \mathrm{kA}$, corresponding to a total stored energy of $130 \mathrm{~J}$. (This represents a global energy confinement time of $W_{p} / P_{E C R H} \approx 26 \mathrm{~ms}$.) When the dipole levitates, the plasma current and stored energy doubles. We also observe thAT levitation doubles the central line-density and improves the stability margin for the hot electron interchange instability. Since measurements of the cyclotron emission, using a $60 \mathrm{GHz}$ radiometer, show only a slightly increased emission during levitation, the doubling of plasma stored energy is attributed to a likely increase thermal electron pressure.

The most dramatic consequence of magnetic levitation of the dipole is the pronounced change in the plasma density profile. The four interferometer cords for the levitated and supported discharges are shown on the right-hand-side of Fig. 3. The differences between levitated and supported operation is striking. When supported, each of the four interferometers show the same line density. When the dipole is levitated, the inner cord is nearly ten times larger than the outer cord, and the line density increases rapidly from the outer edge to the central heating region.

In order to interpret the interferometer measurements, we reconstruct the radial profile of the density using three techniques that produce equivalent results. These are: (i) assume the density varies as $1 / V$ within each of the axisymmetric regions detected by the microwave receivers, (ii) Abel inversion by assuming the interferometer phase varies smoothly between the four channels, and (iii) a profile parameter, called $\Delta$, that relates the mean-squared difference of the ratios of interferometer cords to those rations that would results from density profiles that scale like $n \propto 1 / V^{\alpha}$. These 
reconstructions show that plasma density to be highly peaked when the dipole is levitated and approximately uniform when supported. Furthermore, we find that for the levitated case the assumption of $N=\langle n\rangle V \approx$ constant, appears to describe the plasma density throughout much of the closed-field-line region of the experiment. This observation is highly favorable and strongly supports the dipole fusion concept.

Figure 3 also shows the "profile steepness" for LDX plasma density profiles as a function of the background, neutral pressure. The profile steepness is the exponent $\alpha$ which provides the best-fit in the relationship $n(r) \sim \psi(r)^{\alpha}$, where $n(r)$ is the density profile across the equatorial midplane of the closed-field line region. The density profile steepness of $\alpha \sim 4.5$ corresponds to the linedensity deviation parameter $\Delta \sim 0$. The variation of $\Delta$ with power and neutral gas show strongly peaked profiles in nearly all cases where the dipole is levitated. Remarkably, once the plasmas have sufficient fueling (neutral pressures greater than $5 \mu$ Torr), LDX plasmas tend to have steepness exponents in the narrow range of 3-5. There is no discernible dependence on plasma species and only a slight dependence on the neutral pressure (again, once the plasmas are sufficiently fueled). Plasmas heated with $15 \mathrm{~kW}$ of ECRH have profile steepness exponents very near 4.5 suggesting that these plasmas have very nearly an equal number of particles per flux-tube and can be expected to be stationary to convective interchange mixing. In general the density profiles exhibit much less of a dependence on the experimental conditions than the density magnitudes.

Centrally peaked density profiles are established relatively quickly during levitated discharges. Figure 4 displays temporal variations of the interferometer line-density for two discharges produced with similar conditions except one was produced with a levitated dipole and the other with a supported dipole. During these shots, $12 \mathrm{~kW}$ of multiple-frequency ECRH was applied in short $1.5 \mathrm{~s}$ pulses. During this time the plasma stored energy increased linearly, but remained below a peak $\beta<4 \%$ during the short heating pulse. As shown on the right of Fig. 4, the density for supported and levitated discharges are nearly equal for the first few ms. Large density fluctuations are observed at the edge of supported discharges. In contrast, during magnetic levitation, the density profile continues to steepen, with a rapid inward particle pinch, until a "stationary" profile is reached at $15 \mathrm{~ms}$.

\section{Scrape-of-layer and Global Energy Confinement with Levitation}

The interferometer array shows the peak plasma density to dramatically increase with levitation, but this does not necessarily correspond to an increased particle confinement time. As the plasma density increased in the central region, it often decreased in the outer region relative to the same discharge conditions with a supported dipole. The line densities of LDX plasmas are observed to depend upon four externally-controlled experimental parameters: microwave heating; neutral pressure; plasma species; levitation of the central dipole coil. Importantly, the data shows that by levitating the dipole coil - as opposed to holding it in position with mechanical supports - the line-density increases by as much as a factor of 3 . Furthermore, due to the inward pinch that leads to the stationary profiles observed, the peak density can increase by more than on order of magnitude. These plasmas often exceed the cutoff density of the electron cyclotron waves leading to "overdense" plasmas.

The density in the outer plasma and scrape-off-layer (SOL) has been monitored by swept Langmuir probes. We observe (Fig. 5) that the density in the SOL increases with input power, an indication the the power is utilized to ionize the gas and the resulting plasma flows out along open field lines outside of the magnetic separatrix. The edge temperature remains approximately 


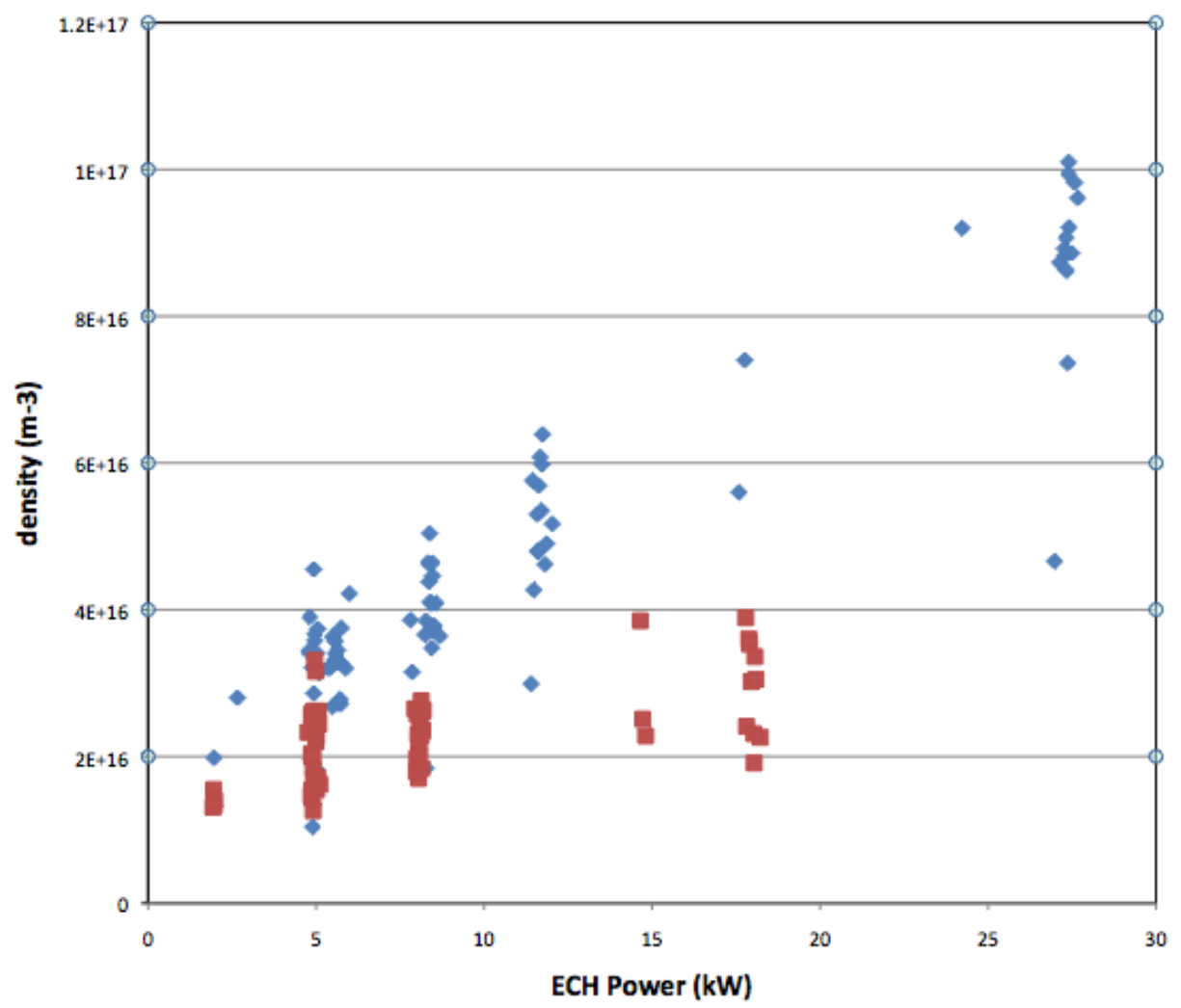

Figure 5: Scrape-off-layer density measured with Langmuir probes during levitation (blue) and during supported operation (red).

constant and therefore the energy confinement time appears to be independent of input power. Figure 5 also includes data from supported discharges. When the dipole is supported a substantial fraction of the plasma flows along field lines to the supports so that the SOL measurements are not indicative the energy loss from the plasma.

\section{Low frequency fluctuations in levitated plasmas}

Plasma created with a levitated dipole have permitted the exploration of the dynamics and transport of plasma confined in a magnetic configuration relevant to a potential dipole fusion reactor. The edge background plasma in LDX has $n_{e} \sim 0.2-5 \times 10^{16} \mathrm{~m}^{-3}$ and $T_{e} \sim 10-20 \mathrm{eV}$ and we observe fluctuations in the diamagnetic drift frequency range $(0.2-10 \mathrm{kHz})$ that are effected by the rate of neutral fueling.

Generally, we find the low-frequency fluctuations to have large spatial structures, low toroidal mode numbers, and to appear with relatively slow temporal dynamics in the plasma's rotating frame of reference [7]. These low-frequency fluctuations co-exist with the high-beta fast electrons but are most probably associated with convective and/or drift-wave-like modes of the cooler background plasma. For typical discharges, low-frequency fluctuations are always observed and may indicate the nature of the plasma electrostatic interchange-like convection that is responsible for peaked profiles with $n \propto V^{-1}$.

While the characteristics of low-frequency fluctuations change during levitation in ways still 
under investigation, levitation improves the stabilization of the gyrokinetic hot electron interchange mode. The hot electron species can be unstable to the hot electron interchange (HEI) [8, 9, 10] mode when the hot electron density gradient is sufficiently high and the background density is sufficiently low [1]. Similarly to supported mode operation, when the dipole is levitated the plasma entered a high-density regime in which the HEI was stabilized for sufficient gas fueling. However, the plasma was maintained in the high-density regime when the gas fueling was substantially reduced as compared to the supported mode. In fact, we have not yet observed a case in levitated operation when high-beta discharges are subject to fast, large-scale instability. Additionally, limiting HEI activity, where HEI occur but lead to small scale bursts of outward energy transport is seldom observed during dipole levitation. In experiments with $15 \mathrm{~kW}$ of input power, and neutral pressure below $1 \mu$ Torr, we have caused intermittent bursts of HEI. We believe the enhanced stability of the HEI during levitation results from both a radial transport broadened hot electron profile and a higher core plasma density resulting from improved particle confinement.

Density "transitions" are induced in LDX plasmas when the ECRH power sources are modulated. In these cases, we have observed a low-frequency fluctuations associated with the rearrange-

ment of the density and the re-establishment of density profiles that more closely approximates the form predicted to be stationary to interchange modes.

\section{Bibliography and References Cited}

\section{LDX Supported Publications}

1. "Fluctuation driven transport and stationary profiles", J. Kesner, D.T. Garnier, M.E. Mauel, Physics of Plasmas, 18, (2011) 050703.

2. "Millimeter-wave radiometer diagnostics of harmonic electron cyclotron emission in the Levitated Dipole Experiment", P.P. Woskov, J. Kesner, D.T. Garnier, M.E.Mauel, Review of Scientific Instruments, 81, (2010).

3. "28 GHz gyrotron ECRH on LDX", P.P. Woskov, J. Kesner, P. Michael, D.T. Garnier, M.E. Mauel, M.E. Journal of Fusion Energy, 29, (2010) 588.

4. "Stationary density profiles in the levitated dipole experiment: toward fusion without tritium fuel", J. Kesner, M.S. Davis, J.L. Ellsworth, D.T. Garnier, J. Kahn, M.E. Mauel, P. Michael, B. Wilson, P.P. Woskov, Plasma Physics and Controlled Fusion, 52, (2012) 124036.

5. "Confinement Improvement with Magnetic Levitation of Superconducting Dipole," D. T. Garnier, A.C. Boxer, J.L. Ellsworth, J.Kesner, and M. E. Mauel, Nuclear Fusion, 49 (2009) 055023 .

6. "Multichannel microwave interferometer for the levitated dipole experiment," Boxer A, Garnier D, Mauel M, Rev Sci Instrum, 80 (2009) 043502.

7. "Stabilization of Low Frequency Instability in a Dipole Plasma," D. T. Garnier, A. K. Hansen, M. E. Mauel, E. E. Ortiz, A. C. Bozer, J. L. Ellsworth, I. Karim, and J. Kesner, J. Plasma Phys, 74 (2008) 737.

8. "Equilibrium Reconstruction of Anisotropic Pressure Profile in the Levitated Dipole Experiment," I. Karin, M. Mauel, J. Ellsworth, A. Boxer, D. Garnier, A. Hansen, J. Kesner, E. Ortiz, J. Fusion Energy, 26 (2007) 99. 
9. "Effects of the Hot Electron Interchange Instability on a Plasma Confined in a Dipolar Magnetic Field," E. Ortiz, A. Boxer, J. Ellsworth, D. Garnier, A. Hansen, J. Kesner, M. Mauel, J. Fusion Energy, 26 (2007) 139.

10. "Varying Electron Cyclotron Resonance Heating on the Levitated Dipole Experiment," A. Hansen, A. Boxer, J. Ellsworth, D. Garnier, J. Kesner, M. Mauel, E. Ortiz, J. Fusion Energy, 26 (2007) 57.

11. "Equilibrium beta limits in a dipole confiuration." L. Guazzotto, J. P. Freidberg and J. Kesner, Phys Plasmas (2007) vol. 14 (6) pp. 062501

12. "Quasilinear theory of interchange modes in a closed field line configuration." A. Kouznetsov, J. P. Freidberg and J. Kesner. Phys Plasmas (2007) vol. 14 (10) pp. 102501

13. "Quench detection for the levitated dipole experiment (LDX) charging coil," P. C. Michael and D. T. Garnier and A. Radovinsky and I. Rodin and V. Ivkin and M. E. Mauel and V. Korsunsky and S. Egorov and A. Zhukovsky and J. Kesner, IEEE Trans. Appl. Supercond. 17 (2007) 2482.

14. "Design and initial operation of the LDX facility," Garnier, D.T., Hansen, A.K.; Kesner, J.; Mauel, M.E.; Michael, P.C.; Minervini, J.V.; Radovinsky, A.; Zhukovsky, A.; Boxer, A.; Ellsworth, J.L.; Karim, I.; Ortiz, E.E. Fusion Engineering and Design, 81, (2006), p 2371-2380

15. "Production and Study of High-Beta Plasma Confined by a Superconducting Dipole Magnet," Garnier, A. Hansen, M. Mauel, E. Ortiz, A. Boxer, I. Karin, J. Kesner, S. Mahar, A. Roach, Physics of Plasmas, 13 (2006) 056111.

16. "First integrated test of the superconducting magnet systems for the Levitated Dipole Experiment (LDX)", Zhukovsky, A., Michael, P.C.; Schultz, J.H.; Smith, B.A.; Minervini, J.V.; Kesner, J.; Radovinsky, A.; Garnier, D.; Mauel, M. Fusion Engineering and Design, 75-79, (2005), p 29-32

17. "Magnetic field perturbations in closed-field-line systems with zero toroidal magnetic field," D. Ryutov, J. Kesner, M. Mauel, Phys. Plasmas 11, (2004) 2318.

18. "Helium Catalyzed D-D Fusion in a Levitated Dipole," J. Kesner, D. Garnier, A. Hansen, M. Mauel, L. Bromberg, Nuc. Fusion 44, 193 (2004).

19. "Advanced technology paths to global climate stability: Energy for a greenhouse planet," Hoffert, MI, Caldeira, K, Benford, G, Criswell, DR, Green, C, Herzog, H, Jain, AK, Kheshgi, HS, Lackner, KS, Lewis, JS, Lightfoot, HD, Manheimer, W, Mankins, JC, Mauel, ME, Perkins, LJ, Schlesinger, ME, Volk, T, Wigley, TML, Science, pp. 981-987, 298 (2002)

20. "Status of the floating coil of the levitated dipole experiment," Zhukovsky, A, Garnier, D, Gung, C, Kesner, J, Mauel, M, Michael, P, Minervini, J, Morgan, M, Pedersen, TS, Radovinsky, A, Schultz, J, IEEE Transactions On Applied Superconductivity, p. 666-669 4 (2002).

21. "Design, fabrication and test of the react and wind, Nb3Sn, LDX floating coil," Smith, B.A.; Schultz, J.H.; Zhukovsky, A.; Radovinsky, A.; Gung, C.; Michael, P.C.; Minervini, J.V.; Kesner, J.; Garnier, D.; Mauel, M.; Naumovich, G.; Kocher, R., IEEE Trans. Applied Superconductivity 11(2001) 2010. 
22. "High temperature superconducting levitation coil for the Levitated Dipole Experiment (LDX)," Schultz, J.H.; Driscoll, G.; Garnier, D.; Kesner, J.; Mauel, M.; Minervini, J.V.; Smith, B.; Radovinsky, A.; Snitchler, G.; Zhukovsky, A., IEEE Trans. Applied Superconductivity 11 (2001) 2004.

23. "Dipole Equilibrium And Stability," Kesner, J. and Simakov, A.N. and Garnier, D.T. and Catto, P.J. and Hastie, R.J. and Krasheninnikov, S.I. and Mauel, M.E. and Sunn Pedersen, T. and Ramos, J.J., Nuclear Fusion, 41 (2001) pp. 301-8.

24. "Dipole equilibrium and stability." Kesner, J., Simakov, A.N., Garnier, D.T., Catto, P.J., Hastie, R.J., Krasheninnikov, S.I., Mauel, M.E., Sunn Pedersen, T., Ramos, J.J., Nucl Fusion (2001) vol. 41 (3) pp. 301-308

25. "Interchange modes in a collisional plasma." Kesner, Phys Plasmas (2000) vol. 7 (10) pp. 3837

26. "Convective cell formation in a levitated dipole." Kesner and Garnier. Phys Plasmas (2000) vol. 7 (6) pp. 2733-2737

27. "MHD Stability in a Levitated Dipole," D. Garnier, J. Kesner, M. Mauel, Phys. Plasmas 6 (1999) 3431.

28. "The Levitated Dipole Experiment (LDX) magnet system," Schultz, J.H. and Kesner, J. and Minervini, J.V. and Radovinsky, A. and Pourrahimi, S. and Smith, B. and Thomas, P. and Wang, P.W. and Zhukovsky, A. and Myatt, R.L. and Kochan, S. and Mauel, M. and Garnier, D., IEEE Transactions on Applied Superconductivity 9 (1999) 378.

29. "Stability of a plasma confined in a dipole field." Kesner, Phys Plasmas (1998) vol. 5 (10) pp. 3675-3679

30. "Plasma Confinement in a Levitated Magnetic Dipole." Kesner and Mauel, Plasma Physics Reports c/c of Fizika Plazmy (1997) vol. 23 pp. 801.

31. "Stability of electrostatic modes in a levitated dipole." Kesner, Phys Plasmas (1997) vol. 4 (2) pp. 419-422

\section{Dissertations}

1. J.L. Ellsworth, "X-Ray Diagnostics for the Levitated Dipole Experiment", M.S., MIT (2004).

2. S.B. Mahar,, "Multiple Frequency Electron Cyclotron Heating for the Levitated Dipole Experiment", M.S., MIT (2005).

3. E.E. Ortiz, "Observation of Hot Electron Interchange Instability in a High Beta Dipole Confined Plasma", Ph.D. Columbia (2007)

4. I. Karim, "Equilibrium and Stability Studies of Plasmas in a Dipole Magnetic Fields Using Magnetic Measurements", Ph.D., MIT, (2007)

5. A.C. Boxer, "Interchange Stationary Profiles in the Levitated Dipole Experiment (LDX)", Ph.D., MIT, (2008) 
6. R. Bergmann, "Characterization of low-frequency electric potential oscillations near the edge of a plasma confined by a levitated magnetic dipole", M.S., MIT, (2009)

7. J.L. Ellsworth, "Characterization of low-frequency density uctuations in dipole-con ned laboratory plasmas", Ph.D. MIT, (2010).

8. M.S. Davis, "Measurement and modeling of the electron pressure profiles in the Levitated Dipole Experiment (LDX)", Ph.D. in preparation, Columbia, (2013).

\section{Bibliography}

[1] Darren T. Garnier, A. K. Hansen, Michael E. Mauel, E. Ortiz, Alexander C. Boxer, Jennifer L Ellsworth, I Karim, Jay Kesner, S Mahar, and A Roach. "production and study of high-beta plasma confined by a superconducting dipole magnet". Phys. Plasmas, 13:056111, Jan 2006.

[2] Carpenter, D.L. and Anderson, R.R. "An ISEE/Whistler Model of Equatorial Electron Density in the Magneto sphere". Journal of Geophysical Research,, Vol. 97, No. A2, pp. 1097-1108, February, 1992.

[3] Burch, J.L.; Mitchell, D.G.; Sandel, B.R.; Brandt, P.C.; Wuest, M. "Global dynamics of the plasmasphere and ring current during magnetic storms". Geophysical Research Letter, v 28, n 6, p 1159-62, 15 March 2001.

[4] Wolf, R.A.; Spiro, R.W.; Sazykin, S.; Toffoletto, F.R. "How the Earth's inner magnetosphere works: An evolving picture". Journal of Atmospheric and Solar-Terrestrial Physics, v 69, n 3, p 288-302, March 2007.

[5] Boxer, A., Garnier, D., Mauel, M. "Multichannel, Microwave Interferometer for the Levitated Dipole Experiment". Review of Scientific Instruments, V. 80, Issue 4 DOI: 10.1063/1.3095684, 2009 .

[6] Gentle, K., Huang, He. "Texas Helimak". Plasma Science and Technology, Vol. 10, June, 2008 .

[7] D.T. Garnier, R.M. Bergmann, A.C. Boxer, J.L. Ellsworth, J. Kesner and M.E. Mauel. "Confinement Improvement with Magnetic Levitation of Superconducting Dipole". NUC FUSION,, v 49 (5)055023, 2009.

[8] N. Krall. Phys. Fluids, V. 9, p. 820, 1966.

[9] B. Levitt, D. Mastovsky and M. Mauel. Phys. Plasmas, 9, p. 2507, 2002.

[10] N. Krasheninnikova, P. Catto. Phys. Plasmas, 12, p. 32101, 2005. 